\title{
Oral Health Knowledge, Awareness and Associated Practices of Pre-school Children's Parents in Damascus, Syria: a cross-sectional study.
}

Sana Lala ( $\square$ sana.lala@hotmail.com )

Damascus University https://orcid.org/0000-0003-1524-1344

\section{Saleh Al Kurdi}

Damascus University

Chaza Kouchaji

Damascus University

Research article

Keywords: Oral hygiene practices, Pre-school children, Parental education level, Child Gender, Child Order, Deleterious Oral Habits

Posted Date: July 8th, 2020

DOI: https://doi.org/10.21203/rs.3.rs-32897/v1

License: (c) (i) This work is licensed under a Creative Commons Attribution 4.0 International License. Read Full License 


\section{Abstract}

Background: Oral health hygiene and practices of pre-school children depends on the knowledge, awareness, and attitude of their parents. Parental education level, family background and family size play an important role in adapting oral hygiene practices. Also, oral health behaviors vary between boys and girls, and it is generally believed that girls are better at taking care of their oral hygiene than boys. This cross-sectional study aimed to assess oral health hygiene and practices of pre-school children (4-6 years old) and its correlation with their parent's education level, child gender and child order between his/her brothers in the family.

Methods: A survey was conducted randomly among 270 parents of Damascus population. Access to the parents in the target age group was achieved through face-to-face interaction (14 parents), online (87 parents) and two different kindergartens in two different social areas (169 parents). A set of 17 questions were formulated, and the questionnaire was distributed. A comparison of the answers from the collected data was made on SPSS 24 using Chi-Square Tests.

Results: Chi-Square Tests showed the important role of parental education level and its associated with regular dental visit $(9.3 \%)$, temporary teeth treatment (48.1\%), no early extracted teeth due to caries $(48.5 \%)$ and no current caries (35.2\%). On the other hand, there was no difference between child gender or order and daily oral hygiene practices.

Conclusion: This study highlights the role of Parental education level in the quality of a child's oral hygiene practices. Although some parents were aware of the importance of temporary teeth treatment and preventing caries through a regular dental visit, they weren't aware of some deleterious oral habits.

\section{Background}

The dental health of preschool children has extensive implications on the oral health of the individual as he grows into an adult. Parents/guardians of preschool children play a central role in enforcing proper oral hygiene and preventive regime in these children.

The oral health care provided by the parents to the pre-school children is of crucial importance here as this determines not only the current oral health status of the child but also lays the backbone of attitudes and practices that a child adopts in this age which he carries over in his or her adulthood.[1] Improvement in children's oral health depends on parent's awareness and knowledge. It is essential to start basic good oral health habits from childhood so that the important dental norms are formed and then maintained into future.[2] Family background plays an important role in adapting oral hygiene practices. [3] Oral health behaviors vary between boys and girls, and it is generally believed that girls have better COHB than boys. $[4,5]$

Dental caries is a multifactorial disease, with many risk factors contributing to their initiation and progression. 
Early childhood caries (ECC) is a term used to describe dental caries in children aged 6 years old. Oral streptococci are considered to be the main etiological agent of tooth decay in children. The risk factors can be categorized as biological, environmental or socio-behavioral [6]. In preschoolers, high consumption of sucrose, sweet drinks, high sugar intake between meals, and frequent snacking have all been associated with dental caries $[7,8]$. Additionally, the quality of a child's oral hygiene practices and the parents' ability to withhold cariogenic snacks are also factors associated with dental caries $[6,9]$. Some studies have found an association between tooth-brushing and lower caries prevalence, although the findings are inconsistent. $[8,10,11]$ Moreover, socioeconomic factors such as income, education level and family size impact disease prevalence. $[12,13]$

Primary teeth play an important role in basic life functions such as speech, phonetics, and eating. The management of deciduous teeth is not considered a primary concern in most of the population. The most increasing problem nowadays is the increase in caries risk referred to as early childhood caries. Further, the treatment of primary teeth is not considered important as it is believed that primary teeth will shed as the child grows, without having an effect on permanent dentition.

Dental caries in children is rapidly increasing compared to that seen in permanent teeth. In early childhood caries, there is an aggressive spread of dental caries most commonly affecting the upper anterior teeth as they are the first teeth to erupt. An increase in dental caries increases the pain experienced by the child, and eventually decreases the intake of food, thereby decreasing the essential minerals and vitamins leading to malnutrition.[14] Early childhood caries is commonly seen in bottlefeeding children overnight because of the increase in sucrose content attacking the tooth surface, thereby increasing caries incidence.

Loss of anterior teeth in children eventually results in speech development and lack of confidence due to peer influence. The space present in the primary dentition due to early extraction must be maintained to prevent unorganized eruption of the permanent teeth. Improper maintenance of space in primary dentition leads to lack of space for eruption of the permanent teeth, leading to crowding and impaction.

Poor oral habits include a wide spectrum of habits including, thumb sucking, finger sucking, blanket sucking, tongue sucking, soother/pacifier use, lip sucking, lip licking, mouth breathing, and nail biting, among others.

These habits can alter the normal muscle balance in the face, resulting in an orofacial myofunctional disorder, which can have a negative impact on facial growth. Thumb sucking is the most recognized oral habit that is widely understood to negatively affect the growth of the jaws and the teeth. When the thumb is in the mouth it displaces the tongue so it is not resting fully in the palate. Lip sucking or lip biting can also have effects on the upper anterior causing proclination. Bruxism is a sleep disorder wherein the child bites his teeth during sleep. Habitual mouth breathing is when the child has a habit of breathing from the mouth instead of the nose. This could be because of three reasons - obstruction in the nasal passage, habitual, or anatomical. These habits require an orthodontic treatment approach, with the use of habit breaking appliances. 
Finger sucking, blanket sucking, soother use, mouth breathing and other poor oral habits can displace the tongue from its normal resting position in the palate with associated negative affects on facial growth.

This cross-sectional study aimed to assess oral health hygiene and practices of pre-school children (46 years old) and its correlation with their parent's education level, child gender and child order between his/her brothers in the family. All data generated or analyzed during this study are included in this published article.

\section{Methods}

\section{Research methodology:}

A cross-sectional research was carried out to study the role of parental education level, child gender and order in daily oral hygiene practices among pre-school children in Damascus, Syria.

\section{Sampling:}

The data for this cross-sectional study were collected in March 2020. The target population was parents having children between 4-6 years of age. Written parental consent was obtained for this study. This age group was chosen because children depend on their parents to have good dental health practices. In this age, it's considered that it is the parent's responsibility to make these practices as a habit in their children.

\section{Survey's Questions:}

A survey (Supplementary file 1) was conducted randomly among 270 parents of Damascus population. Access to the parents in the target age group was achieved through face-to-face interaction (14 parents), online (87 parents) and two different kindergartens in two different social areas (169 parents). A set of 17 questions was formulated, and the questionnaire was distributed. The questionnaire collected information about the (e.g., paternal education levels, child gender and order) and dental health practices (e.g., details about tooth-brushing duration and frequency, toothpaste amount used and toothbrush changing period). The questionnaire also asked about eating habits (e.g., sugar and soda intake frequency), deleterious oral habits (e.g., thumb sucking, nail and lip biting, mouth breathing, bruxism, tongue thrusting...), dental visit, current caries, the way of handling orthodontic problems and early tooth extraction due to caries.

\section{Statistical analysis:}

The information was collected and recorded. A comparison of the answers from the collected data was made on SPSS 24 using Chi-Square Tests.

\section{Results}

General Study Results:

Results showed that $150(55.6 \%)$ of the parents are educated and $120(44.4 \%)$ are not. $137(50.7 \%)$ of the children are males and $133(49.3 \%)$ are females. 122 (41.5\%) children of them are the oldest between 
their brothers and sisters whereas $39(14.4 \%)$ are midchilds and $119(44.1 \%)$ are the youngest. (Table 1$)$

It's found that daily tooth-brushing frequency was zero in $4.8 \%$ of the children. And $62.2 \%$ of them brush their teeth just once a day. $31.5 \%$ of them brush their teeth twice a day and only $1.5 \%$ brush their teeth three times a day. Also, it's found that tooth-brush duration was only one minute in $38.1 \%, 2$ minutes in $35.9 \%, 3$ minutes only in $13.3 \%$ and less than one minute in $12.6 \%$. On the other hand, it's found that toothpaste amount used was as rice size in only $15.2 \%$ and it was as peas size in $75.9 \%$, less than rice size in $2.6 \%$ and more than peas size in $6.3 \%$. The results showed that the duration of using toothbrush before changing it was 6 months in $57.0 \%$, one year in $17.0 \%$, less than 6 months in $21.1 \%$ and more than one year in $4.8 \%$. (Table 2 )

Table 2

Daily brushing frequency, brushing duration, toothpaste amount and toothbrush changing period.

\begin{tabular}{|llc|}
\hline Variables & $\mathbf{N}=\mathbf{2 7 0}$ \\
\hline \multicolumn{1}{|c|}{$\mathbf{n}$} & $\%$ \\
\hline Parental education level & & \\
\hline Educated & 150 & 55.6 \\
\hline Uneducated & 120 & 44.4 \\
\hline Child Gender & & \\
\hline Male & 137 & 50.7 \\
\hline Female & 133 & 49.3 \\
\hline Child Order & & \\
\hline Oldest & 112 & 41.5 \\
\hline Midchild & 39 & 14.4 \\
\hline Youngest & 119 & 44.1 \\
\hline
\end{tabular}

About the dental visit, it was regularly only in $13.3 \%$ whereas $62.2 \%$ of the dental visit was only when the child complains of pain and $24.4 \%$ never taken to a dentist. Only $9.6 \%$ of the parents thought that treatment of primary tooth is unimportant whereas $90.4 \%$ thought that dental treatment in children was necessary. When the parents were asked about the way of handling any orthodontic problems $45 \%$ of them suggested that they will wait until the permanent dentition whereas $54.1 \%$ suggested that they will have an orthodontic consulted. Only $9.639 \%$ of children had applied local fluoride on their teeth whereas $90.370 \%$ did not. (Table 3 ) 
Table 3

Dental visit, temporary tooth treatment, handling orthodontic problems and applies local fluoride.

\begin{tabular}{|lll|}
\hline Variables & \multicolumn{2}{l|}{$\mathbf{N}=\mathbf{2 7 0}$} \\
\hline & \multicolumn{1}{l|}{$\%$} \\
\hline Daily Brushing Frequency & & \\
\hline 0 & 13 & 4.8 \\
\hline 1 & 168 & 62.2 \\
\hline 2 & 85 & 31.5 \\
\hline 3 & 4 & 1.5 \\
\hline Brushing Duration & & \\
\hline 1 minute & 103 & 38.1 \\
\hline 2 minutes & 97 & 35.9 \\
\hline 3 minutes & 36 & 13.3 \\
\hline Less & 34 & 12.6 \\
\hline Toothpaste Amount & & \\
\hline Rice & 41 & 15.2 \\
\hline Peas & 205 & 75.9 \\
\hline More & 17 & 6.3 \\
\hline Less & 7 & 2.6 \\
\hline Toothbrush Changing Periods & & \\
\hline 6 months & 154 & 57.0 \\
\hline a year & 46 & 17.0 \\
\hline More & 13 & 4.8 \\
\hline Less & 57 & 21.1 \\
\hline
\end{tabular}

Sugar daily intake was zero in $1.1 \%$, once a day in $25.9 \%$, twice a day in 49.6 , three times a day in $23.3 \%$. On the other hand, Soda weekly intake was zero in $68.9 \%$, once a week in $25.2 \%$, twice a week in $4.4 \%, 3$ times in $1.5 \%$. (Table 4) 
Table 4

Sugar daily intake and soda weekly intake.

\begin{tabular}{|llc|}
\hline Variables & $\mathbf{N}=\mathbf{2 7 0}$ \\
\hline & $\mathbf{n}$ & $\%$ \\
\hline Dental Visit & & \\
\hline Regularly & 36 & 13.3 \\
\hline On pain & 168 & 62.2 \\
\hline Never & 66 & 24.4 \\
\hline Temporary Tooth Treatment & & \\
\hline Important & 244 & 90.4 \\
\hline Not important & 26 & 9.6 \\
\hline Handling Orthodontic problems & & \\
\hline Permanent waiting & 124 & 45.9 \\
\hline Orthodontist consulted & 146 & 54.1 \\
\hline Applies Local Fluoride & & \\
\hline Done & 26 & 9.6 \\
\hline Never & 244 & 90.4 \\
\hline
\end{tabular}

It's found that $45.6 \%$ of children have current caries, $26.8 \%$ of them on anterior teeth whereas $73.2 \%$ where on posterior teeth. On the other hand, $54.4 \%$ of children don't have any current caries. Also, it's found that $83 \%$ of children don't have any early extraction due to caries whereas there are $5.9 \%$ of children had extracted one tooth, $4.4 \%$ had extracted 2 teeth, $3 \%$ had extracted 3 teeth, $3.3 \%$ had extracted 4 and only $0.4 \%$ had extracted more than four teeth because of caries. (Table 5 ) 
Table 5

Current caries, if caries positive and

extracted or lost teeth due to caries.

\begin{tabular}{|lll|}
\hline Variables & $\mathbf{N}=\mathbf{2 7 0}$ \\
\hline & $\mathbf{n}$ & $\%$ \\
\hline Sugar Daily Intake & & \\
\hline 0 & 3 & 1.1 \\
\hline 1 & 70 & 25.9 \\
\hline 2 & 134 & 49.6 \\
\hline 3 & 63 & 23.3 \\
\hline Soda Weekly Intake & & \\
\hline 0 & 186 & 68.9 \\
\hline 1 & 68 & 25.2 \\
\hline 2 & 12 & 4.4 \\
\hline 3 & 4 & 1.5 \\
\hline
\end{tabular}

Finally, it's noticed that the practices of deleterious oral habits are widespread, $15.556 \%$ of the children have nails biting, $12.592 \%$ have mouth breathing, $12.962 \%$ have bruxism, $11.481 \%$ of children put things inside their mouths, $6.296 \%$ have thumb sucking, $5.185 \%$ have lips biting, $2.22 \%$ have tongue thrusting, $0.370 \%$ have bottle-feeding, $0.740 \%$ have food sucking whereas $45.55 \%$ of children don't have any deleterious oral habits. (Fig. 1)

\section{Statistical Analysis using Chi-Square Tests:}

- Parental Education Level:

The test showed a statistically significant difference between parental education level and dental visit, temporary teeth treatment, early extracted teeth due to caries, current caries. (Table 6) The dental visit was more regularly in children with educated parents, while taking children to the dentist only when they complain of pain was more in uneducated parents. More children with educated parents had never taken to the dentist before. (Table 6) More educated parents think that the treatment of temporary teeth is important. Whereas more uneducated parents think that it is not important. (Table 6) Children with educated parents had less extracted tooth due to caries than children with uneducated parents that had more extracted tooth. (Table 6) Children with educated parents had fewer caries than uneducated parents. (Table 6) 
Table 6

Comparison between parental education level and some oral hygiene practices.

\begin{tabular}{|c|c|c|}
\hline \multirow[t]{2}{*}{ Variables } & \multicolumn{2}{|c|}{$N=270$} \\
\hline & $\mathrm{n}$ & $\%$ \\
\hline \multicolumn{3}{|c|}{ Current Caries } \\
\hline Yes & 123 & 45.6 \\
\hline No & 147 & 54.4 \\
\hline \multicolumn{3}{|c|}{ If Caries Positive } \\
\hline On Anterior & 33 & 26.8 \\
\hline On Posterior & 90 & 73.2 \\
\hline \multicolumn{3}{|c|}{ Extracted or Lost teeth due to Caries } \\
\hline 0 & 224 & 83.0 \\
\hline 1 & 16 & 5.9 \\
\hline 2 & 12 & 4.4 \\
\hline 3 & 8 & 3.0 \\
\hline 4 & 9 & 3.3 \\
\hline 6 & 1 & .4 \\
\hline
\end{tabular}

Statistically there was no significant difference between parental education level and daily brushing frequency, brushing duration, toothpaste amount, toothbrush changing periods, applying local fluoride, the way of handling orthodontic problems and sauger and soda intake. (Table 7) 
Table 7

Comparison between parental education level and some oral hygiene practices.

\begin{tabular}{|c|c|c|c|c|c|}
\hline \multirow[t]{3}{*}{ Variable } & \multicolumn{4}{|c|}{ Parental Educational level } & \multirow[t]{3}{*}{$P$ value } \\
\hline & \multicolumn{2}{|c|}{$\begin{array}{l}\text { Educated } \\
(\mathrm{N}=150)\end{array}$} & \multicolumn{2}{|c|}{$\begin{array}{l}\text { Uneducated } \\
(\mathrm{N}=120)\end{array}$} & \\
\hline & $\mathbf{N}$ & $\%$ & $\mathbf{N}$ & $\%$ & \\
\hline \multicolumn{6}{|l|}{ Dental visit } \\
\hline Regularly & 25 & 69.45 & 11 & 30.55 & $.001 *$ \\
\hline On pain & 79 & 47.02 & 89 & 52.98 & \\
\hline Never & 46 & 69.7 & 20 & 30.3 & \\
\hline \multicolumn{6}{|c|}{ Temporary teeth treatment } \\
\hline Important & 130 & 53.28 & 114 & 46.72 & $.023^{\star}$ \\
\hline Not important & 20 & 76.92 & 6 & 23.08 & \\
\hline \multicolumn{6}{|c|}{ Extracted Or lost teeth } \\
\hline 0 & 131 & 58.49 & 93 & 41.51 & $.002 * \square$ \\
\hline 1 & 8 & 50.00 & 8 & 50.00 & \\
\hline 2 & 8 & 66.67 & 4 & 33.33 & \\
\hline 3 & 2 & 25.00 & 6 & 75.00 & \\
\hline 4 & 0 & 0 & 9 & 100.0 & \\
\hline 6 & 1 & 100.0 & 0.00 & 0.00 & \\
\hline \multicolumn{6}{|l|}{ Current Caries } \\
\hline Yes & 55 & 44.71 & 68 & 55.29 & $.001^{\star}$ \\
\hline No & 95 & 64.62 & 52 & 35.38 & \\
\hline
\end{tabular}

- Child Gender:

Statically there was no significant difference between child gender and daily brush frequency, brushing duration nor toothpaste amount, toothbrush changing periods, dental visit, the importance of temporary teeth treatment, local fluoride appliance, the way of handling orthodontic problems, sugar and soda intake, the number of extracted teeth due to caries and current caries. (Table 8) 
Table 8

No statistical difference between child gender and oral hygiene practices.

\begin{tabular}{|c|c|c|c|c|c|}
\hline \multirow[t]{3}{*}{ Variable } & \multicolumn{4}{|c|}{ Parental Educational level } & \multirow[t]{3}{*}{$P$ value } \\
\hline & \multicolumn{2}{|c|}{$\begin{array}{l}\text { Educated } \\
(\mathrm{N}=150)\end{array}$} & \multicolumn{2}{|c|}{$\begin{array}{l}\text { Uneducated } \\
(\mathrm{N}=120)\end{array}$} & \\
\hline & $\mathbf{N}$ & $\%$ & $\mathbf{N}$ & $\%$ & \\
\hline \multicolumn{6}{|c|}{ Daily brushing frequency } \\
\hline 0 & 7 & 53.84 & 6 & 46.16 & $.54 \rrbracket$ \\
\hline 1 & 100 & 59.53 & 68 & 40.47 & \\
\hline 2 & 39 & 45.88 & 46 & 54.12 & \\
\hline 3 & 4 & 100.0 & 0 & 0.00 & \\
\hline \multicolumn{6}{|c|}{ Brushing Duration } \\
\hline 1 minute & 60 & 58.25 & 43 & 41.75 & .591 \\
\hline 2 minutes & 56 & 57.73 & 41 & 42.27 & \\
\hline 3 minutes & 17 & 47.22 & 19 & 52.78 & \\
\hline Less & 17 & 50.00 & 17 & 50.00 & \\
\hline \multicolumn{6}{|c|}{ Toothpaste Amount } \\
\hline Rice & 28 & 68.29 & 13 & 31.71 & $.181 \rrbracket$ \\
\hline Peas & 111 & 54.15 & 94 & 45.85 & \\
\hline More & 9 & 52.94 & 8 & 47.06 & \\
\hline Less & 2 & 28.57 & 5 & 71.43 & \\
\hline \multicolumn{6}{|c|}{ Toothbrush Changing Periods } \\
\hline 6 months & 85 & 55.19 & 69 & 44.81 & .563 \\
\hline a year & 28 & 60.87 & 18 & 39.13 & \\
\hline More & 5 & 38.46 & 8 & 61.54 & \\
\hline Less & 32 & 56.14 & 25 & 43.86 & \\
\hline \multicolumn{6}{|c|}{ Applies Local Fluoride } \\
\hline Done & 19 & 73.07 & 7 & 26.92 & .064 \\
\hline Never & 131 & 53.69 & 113 & 46.31 & \\
\hline
\end{tabular}




\begin{tabular}{|c|c|c|c|c|c|}
\hline \multirow{2}{*}{$\begin{array}{l}\text { Variable } \\
\text { Permanent waiting }\end{array}$} & \multicolumn{4}{|c|}{ Parental Educational level } & \multirow{2}{*}{$\begin{array}{l}\text { P value } \\
1.000\end{array}$} \\
\hline & 69 & 48.59 & 55 & 51.41 & \\
\hline Orthodontist consulted & 81 & 55.48 & 65 & 44.52 & \\
\hline \multicolumn{6}{|l|}{ Sugar Daily Intake } \\
\hline 0 & 1 & 33.33 & 2 & 66.67 & $.897 \rrbracket$ \\
\hline 1 & 39 & 55.71 & 31 & 44.29 & \\
\hline 2 & 74 & 55.22 & 60 & 44.78 & \\
\hline 3 & 36 & 57.14 & 27 & 42.86 & \\
\hline \multicolumn{6}{|l|}{ Soda Weekly Intake } \\
\hline 0 & 109 & 58.60 & 77 & 41.40 & $.198 \rrbracket$ \\
\hline 1 & 36 & 52.94 & 32 & 47.06 & \\
\hline 2 & 4 & 33.33 & 8 & 66.67 & \\
\hline 3 & 1 & 25.00 & 3 & 75.00 & \\
\hline
\end{tabular}

- Child's Order:

Statically there was no significant difference between child order and the rest data (daily brush frequency, brushing duration, toothpaste amount, toothbrush changing periods, dental visit, the importance of temporary teeth treatment, local fluoride appliance, the way of handling orthodontic problems, sugar and soda intake, the number of extracted teeth due to caries and current caries). (Table 9) 
Table 9

Comparison between child order and oral hygiene practices.

\begin{tabular}{|c|c|c|c|c|c|}
\hline \multirow[t]{3}{*}{ Variable } & \multicolumn{4}{|c|}{ Gender } & \multirow{3}{*}{$\begin{array}{l}P \\
\text { value }\end{array}$} \\
\hline & \multicolumn{2}{|c|}{$\begin{array}{l}\text { Male }(\mathrm{N}= \\
137)\end{array}$} & \multicolumn{2}{|c|}{$\begin{array}{l}\text { Female }(N= \\
133)\end{array}$} & \\
\hline & $\mathbf{N}$ & $\%$ & $\mathbf{N}$ & $\%$ & \\
\hline \multicolumn{6}{|c|}{ Daily brushing frequency } \\
\hline 0 & 10 & 76.92 & 3 & 23.08 & $.123 \rrbracket$ \\
\hline 1 & 88 & 52.39 & 80 & 47.61 & \\
\hline 2 & 37 & 43.52 & 48 & 56.48 & \\
\hline 3 & 2 & 50.00 & 2 & 50.00 & \\
\hline \multicolumn{6}{|c|}{ Brushing Duration } \\
\hline 1 minute & 59 & 57.29 & 44 & 42.71 & .404 \\
\hline 2 minutes & 46 & 47.42 & 51 & 52.58 & \\
\hline 3 minutes & 16 & 44.44 & 20 & 55.56 & \\
\hline Less & 16 & 47.06 & 18 & 52.94 & \\
\hline \multicolumn{6}{|c|}{ Toothpaste Amount } \\
\hline Rice & 21 & 51.22 & 20 & 48.78 & $.779 \square$ \\
\hline Peas & 102 & 49.76 & 103 & 50.24 & \\
\hline More & 9 & 52.94 & 8 & 47.06 & \\
\hline Less & 5 & 71.43 & 2 & 28.57 & \\
\hline
\end{tabular}

\section{Toothbrush Changing}

Periods

\begin{tabular}{llllll}
6 months & 75 & 48.70 & 79 & 51.30 & $.053 \rrbracket$ \\
a year & 31 & 67.39 & 15 & 32.61 & \\
More & 4 & 30.77 & 9 & 69.23 \\
\hline Less & 27 & 47.37 & 30 & 52.63
\end{tabular}

\section{Dental Visit}

$\begin{array}{llllll}\text { Regularly } & 19 & 52.78 & 17 & 47.22 & .311 \\ \text { On pain } & 90 & 53.57 & 72 & 46.43 & \end{array}$




\begin{tabular}{|c|c|c|c|c|c|}
\hline \multirow{2}{*}{$\begin{array}{l}\text { Variable } \\
\text { Never }\end{array}$} & \multicolumn{4}{|c|}{ Gender } & \multirow{2}{*}{$\begin{array}{l}P \\
\text { value }\end{array}$} \\
\hline & 28 & 42.42 & 38 & 57.58 & \\
\hline \multicolumn{6}{|l|}{$\begin{array}{l}\text { Temporary Tooth } \\
\text { Treatment }\end{array}$} \\
\hline Important & 128 & 52.46 & 116 & 47.54 & .100 \\
\hline Not important & 9 & 34.62 & 17 & 65.38 & \\
\hline \multicolumn{6}{|l|}{ Applies Local Fluoride } \\
\hline Done & 9 & 34.61 & 17 & 65.39 & .100 \\
\hline Never & 128 & 52.46 & 116 & 47.54 & \\
\hline \multicolumn{6}{|l|}{$\begin{array}{l}\text { Handling Orthodontic } \\
\text { problems }\end{array}$} \\
\hline Permanent waiting & 62 & 50.50 & 62 & 50.50 & .903 \\
\hline orthodontist consulted & 75 & 51.37 & 71 & 48.63 & \\
\hline \multicolumn{6}{|l|}{ Sugar Daily Intake } \\
\hline 0 & 1 & 33.33 & 2 & 66.67 & $.067 \rrbracket$ \\
\hline 1 & 27 & 38.57 & 43 & 61.43 & \\
\hline 2 & 72 & 53.73 & 62 & 46.27 & \\
\hline 3 & 37 & 58.73 & 26 & 41.27 & \\
\hline \multicolumn{6}{|l|}{ Soda Weekly Intake } \\
\hline 0 & 97 & 52.15 & 89 & 47.85 & $.899 \rrbracket$ \\
\hline 1 & 32 & 47.06 & 36 & 52.94 & \\
\hline 2 & 6 & 50.00 & 6 & 50.00 & \\
\hline 3 & 2 & 50.00 & 2 & 50.00 & \\
\hline \multicolumn{6}{|l|}{ Extracted or lost teeth } \\
\hline 0 & 113 & 50.44 & 111 & 49.56 & .8980 \\
\hline 1 & 9 & 56.25 & 7 & 43.75 & \\
\hline 2 & 5 & 41.67 & 7 & 58.33 & \\
\hline 3 & 5 & 62.5 & 3 & 37.5 & \\
\hline 4 & 4 & 44.44 & 5 & 55.56 & \\
\hline
\end{tabular}




\begin{tabular}{|lllllll|}
\hline Variable & \multicolumn{2}{l}{ Gender } & & & $\begin{array}{l}\text { P } \\
\text { value }\end{array}$ \\
\hline 6 & 1 & 100.0 & 0 & 0.00 & \\
\hline Current Caries & & & & & \\
\hline Yes & 67 & 54.47 & 56 & 45.53 & .274 \\
\hline No & 70 & 47.62 & 77 & 52.38 & \\
\hline
\end{tabular}

* Statistical significance $p \leq 0.05$.

口 Fisher's exact test was used as $25 \%$ of cells had expected count less than 5 .

Variable

\begin{tabular}{|c|c|c|}
\hline \multicolumn{3}{|c|}{ Child Order } \\
\hline $\begin{array}{l}\text { Oldest }(\mathrm{N}= \\
112)\end{array}$ & Midchild (N = 39) & $\begin{array}{l}\text { Youngest (N= } \\
119)\end{array}$ \\
\hline$\%$ & $\mathbf{N}$ & $\mathbf{N}$ \\
\hline
\end{tabular}

Daily brushing frequency

\begin{tabular}{|lllllllll|}
\hline 0 & 4 & 30.77 & 2 & 15.38 & 7 & 53.85 & .718凶 \\
\hline 1 & 73 & 43.45 & 26 & 15.47 & 69 & 41.08 & \\
\hline 2 & 33 & 38.82 & 10 & 11.76 & 42 & 49.42 & \\
\hline 3 & 2 & 50.00 & 1 & 25.00 & 1 & 25.00 & \\
\hline Brushing Duration & & & & & & & \\
\hline 1 minute & 47 & 45.63 & 13 & 12.63 & 43 & 41.74 & .579 \\
\hline 2 minutes & 38 & 39.17 & 16 & 16.51 & 43 & 44.32 & \\
\hline 3 minutes & 17 & 47.22 & 3 & 8.34 & 16 & 44.44 & \\
\hline Less & 10 & 29.41 & 7 & 20.59 & 17 & 50.00 & \\
\hline Toothpaste Amount & & & & & & & \\
\hline Rice & 61 & 39.61 & 23 & 14.93 & 70 & 45.46 & $.293 \rrbracket$ \\
\hline Peas & 20 & 43.48 & 5 & 10.87 & 21 & 45.65 & \\
\hline More & 4 & 30.77 & 0 & 0.00 & 9 & 69.23 & \\
\hline Less & 27 & 47.34 & 11 & 19.29 & 19 & 33.37 & \\
\hline
\end{tabular}

\section{Toothbrush Changing}

Periods 


\begin{tabular}{|c|c|c|c|c|c|c|c|}
\hline \multirow{2}{*}{$\begin{array}{l}\text { Variable } \\
6 \text { months }\end{array}$} & \multicolumn{3}{|c|}{ Gender } & \multicolumn{4}{|l|}{$\begin{array}{l}P \\
\text { value }\end{array}$} \\
\hline & 20 & 48.78 & 4 & 9.76 & 17 & 41.46 & $.208 \rrbracket$ \\
\hline a year & 79 & 38.53 & 31 & 15.12 & 95 & 46.35 & \\
\hline More & 10 & 58.82 & 4 & 23.53 & 3 & 17.65 & \\
\hline Less & 3 & 42.85 & 0 & 0.00 & 4 & 57.15 & \\
\hline \multicolumn{8}{|l|}{ Dental Visit } \\
\hline Regularly & 15 & 41.66 & 6 & 16.68 & 15 & 41.66 & .061 \\
\hline On pain & 60 & 35.71 & 28 & 16.67 & 80 & 47.62 & \\
\hline Never & 37 & 56.06 & 5 & 7.58 & 24 & 36.36 & \\
\hline \multicolumn{8}{|l|}{$\begin{array}{l}\text { Temporary Tooth } \\
\text { Treatment }\end{array}$} \\
\hline Important & 103 & 42.21 & 34 & 13.94 & 107 & 43.85 & .695 \\
\hline Not important & 9 & 34.61 & 5 & 19.24 & 12 & 46.15 & \\
\hline \multicolumn{8}{|l|}{ Applies Local Fluoride } \\
\hline Done & 14 & 53.84 & 4 & 15.39 & 8 & 30.77 & $.327 \rrbracket$ \\
\hline Never & 98 & 40.16 & 35 & 14.35 & 111 & 45.49 & \\
\hline \multicolumn{8}{|l|}{$\begin{array}{l}\text { Handling Orthodontic } \\
\text { problems }\end{array}$} \\
\hline Permanent waiting & 48 & 38.71 & 17 & 13.71 & 59 & 47.58 & .585 \\
\hline orthodontist consulted & 64 & 43.83 & 22 & 15.08 & 60 & 41.09 & \\
\hline \multicolumn{8}{|l|}{ Sugar Daily Intake } \\
\hline 0 & 2 & 66.67 & 0 & 0.00 & 1 & 33.33 & $.892 \rrbracket$ \\
\hline 1 & 31 & 44.28 & 9 & 12.87 & 30 & 42.85 & \\
\hline 2 & 53 & 39.55 & 23 & 17.16 & 58 & 43.29 & \\
\hline 3 & 26 & 41.27 & 7 & 11.11 & 30 & 47.62 & \\
\hline \multicolumn{8}{|l|}{ Soda Weekly Intake } \\
\hline 0 & 79 & 42.47 & 29 & 15.59 & 78 & 41.94 & $.889 \bigotimes$ \\
\hline 1 & 27 & 39.50 & 8 & 11.97 & 33 & 48.53 & \\
\hline 2 & 5 & 41.67 & 1 & 8.33 & 6 & 50.0 & \\
\hline
\end{tabular}




\begin{tabular}{|c|c|c|c|c|c|c|c|}
\hline \multirow{2}{*}{$\begin{array}{l}\text { Variable } \\
3\end{array}$} & \multicolumn{3}{|c|}{ Gender } & \multicolumn{4}{|l|}{$\begin{array}{l}\mathrm{P} \\
\text { value }\end{array}$} \\
\hline & 1 & 25.00 & 1 & 25.00 & 2 & 50.00 & \\
\hline \multicolumn{8}{|c|}{ Extracted or lost teeth } \\
\hline 0 & 93 & 41.51 & 32 & 14.30 & 99 & 44.19 & $.924 \rrbracket$ \\
\hline 1 & 7 & 43.75 & 2 & 12.50 & 7 & 43.75 & \\
\hline 2 & 5 & 41.67 & 2 & 16.66 & 5 & 41.67 & \\
\hline 3 & 5 & 62.5 & 1 & 12.50 & 2 & 25.00 & \\
\hline 4 & 2 & 22.22 & 2 & 22.22 & 5 & 55.56 & \\
\hline 6 & 0 & 0.00 & 0 & 0.00 & 1 & 100.0 & \\
\hline \multicolumn{8}{|c|}{ Current Caries } \\
\hline Yes & 48 & 39.02 & 18 & 14.64 & 57 & 46.34 & .760 \\
\hline No & 64 & 43.53 & 21 & 14.30 & 62 & 42.17 & \\
\hline \multicolumn{8}{|c|}{ * Statistical significance $p \leq 0.05$} \\
\hline \multicolumn{8}{|c|}{$\begin{array}{l}\text { Fisher's exact test was used as } 25 \% \text { of cells had expected count less than } \\
5 \text {. }\end{array}$} \\
\hline
\end{tabular}

\section{Discussion}

The child's parents/guardians play an important role in the development of healthy habits in children and sustaining the same during the child's transition into adulthood.[15]

Improvement in children's oral health depends on parent's awareness, knowledge and education level. It is essential to start basic good oral health habits from childhood so that the important dental norms are formed and then maintained into future. Family background plays an important role in adapting oral hygiene practices.[16] The family is the first institution that influences child behavior and development, especially the mothers, who are the primary role model for developing behavior.

A significant association was observed in this study between scores of knowledge, attitude and practice and the educational level of the participants which was in accordance with the studies conducted by Jain et al., Suresh et al. [17] and Williams et al.,[18] wherein it was shown that parents with lower education level had poor dental knowledge and attitude also. Probably, the parents with higher education level had better knowledge regarding the oral health care of their children which resulted in favorable attitudes and adoption of better practices to render oral health care to their child.[19] 
A randomized controlled trial done in the UK showed that mothers visit to trained dental educator (dentist) of those pre-school children at risk of caries increased the parental knowledge and improved the attitude toward the dental health of their offspring's. [20]

The factors responsible for irregular visits and follow-up could vary depending on financial status, fear and lack of awareness and motivation.[19] Despite the fact that majority of parents were aware of the fact that regular dental check-ups are necessary, only $13.3 \%$ of parents took their child for dental visit in every 6 months.

The family's influence on COHB varies between girls and boys [4,5]; however, conclusions regarding these differences are inconsistent among existing studies. In this study, there was no difference between boys and girls.

\section{Conclusion}

From this study, it can be concluded that parental education level plays the most important role in the quality of their children's oral hygiene practices.

Most parents don't have the required knowledge about the correct oral hygiene practices such as the daily brushing frequency, brushing duration, the right toothpaste amount that should be used. Most parents don't know the importance of applying local fluoride on their children's teeth which can be done in the regular dental checkups.

On the other hand, some of them were aware of the bad consequences for deleterious oral habits that can make orthodontic problems, eating habits, the importance of temporary teeth treatment and the correct duration to change their children's toothbrush.

Nowadays there is no differentiation between brothers if they are boys or girls or even the oldest or the youngest. All children take the same dental care from their parents, the same oral hygiene practices. What makes a real difference in the children's oral hygiene practices is their parent's education level. The more they are educated, the more they will be taking care of details such caries, fluoride appliance and regular dental checkup which reduce the number of extracted teeth due to caries followed by orthodontic problems.

\section{Abbreviations}

$\mathrm{N}$

Number. *:Statistical significance $p \leq 0.05$. D:Fisher's exact test was used as $25 \%$ of cells had expected count less than 5 .

\section{Declarations}


Acknowledgements: This research could not be completed without the help of the director of the Damascus Kindergarten and the Al-Burouj Kindergarten.

Ethics approval and consent of participate: The research ethical approval was obtained from the scientific committee at the faculty of Dentistry in February. Written parental consent was obtained for this study.

Consent to publish: Not applicable.

Availability of data and materials: All necessary data are presented within the manuscript. All other materials and data are available upon request. For any more details regarding the data of this research please contact the corresponded author - Dr. Sana Lala.

Competing interests: The authors declared no potential conflicts of interest with respect to the research, authorship and/or publication of this article.

Funding: The authors confirm the independence of this research completely from any governmental or non-governmental authorities or local /international organizations.

Authors' Contributions: SL: Wrote the background, methods, results, discussion and conclusion section, designed the chart. SAK: completed the statistical analysis, helped in finalizing the paper. CK: participated as the research supervisor, edited and checked up the research manuscript. All authors approved the final manuscript as submitted and agreed to be accountable for all aspects of the work.

Acknowledgements: This research could not be completed without the help of Damascus Kindergarten and Al-Borouj Kindergarten.

\section{Author's Information:}

- Sana Lala (Corresponding author)

Sana.Iala@hotmail.com

00963988836656

Damascus University

- Saleh Al Kurdi

Salekh1889@gmail.com

00963948844036

Damascus University

- Chaza Kouchaji 
ch.kouchaji@damasuniv.edu.sy

00963944283890

Damascus University

\section{References}

1. Selwitz RH, Ismail Al, Pitts NB. Dental caries. Lancet. 2007;369.

2. -59. Edelstein BL. The dental caries pandemic and disparities problem. BMC Oral Health. 2006;6(Suppl 1):2.

3. Petersen PE, Bourgeois D, Ogawa H, Estupinan-Day S, Ndiaye C. The global burden of oral diseases and risks to oral health. Bull World Health Organ. 2005;83.

4. -669 .

5. Poutanen R, Lahti S, Tolvanen M, et al. Gender differences in child-related and parent-related determinants of oral health-related lifestyle among 11- to 12-year-old Finnish schoolchildren Acta Odontol Scand. 2007;65.

6. -200 .

7. Poutanen R, Lahti S, Tolvanen M, et al. Gender differences in child-related and parent-related determinants of oral health-related lifestyle among 11- to 12-year-old Finnish schoolchildren. Acta Odontol Scand. 2007;65.

8. -200 .

9. Ostberg AL, Halling A, Lindblad U. Gender differences in knowledge, attitude, behavior and perceived oral health among adolescents. Acta Odontol Scand. 1999;57.

10. -236 .

11. Selwitz RH, Ismail Al, Pitts NB. Dental caries. Lancet. 2007;369.

12. -59 .

13. Kowash MB. Severity of early childhood caries in preschool children attending Al-Ain dental Centre, United Arab Emirates. Eur Arch Paediatr Dent. 2015;16.

14. -324 .

15. Kowash MB, Alkhabuli JO, Dafaalla SA, Shah A, Khamis AH. Early childhood caries and associated risk factors among preschool children in Ras Al-Khaimah, United Arab Emirates. Eur Arch Paediatr Dent. 2017;18.

16. -103 .

17. Hong CH, Bagramian RA, Hashim Nainar SM, Straffon LH, Shen L, Hsu CY. High caries prevalence and risk factors among young preschool children in an urban community with water fluoridation. Int J Paediatr Dent. 2014;24.

18. -42 . 
19. Elidrissi SM, Naidoo S. Prevalence of dental caries and toothbrushing habits among preschool children in Khartoum state, Sudan. Int Dent J. 2016;66.

20. -220 .

21. Chedid NR, Bourgeois D, Kaloustian H, Baba NZ, Pilipili C. Caries prevalence and caries risk in a sample of Lebanese preschool children. Odontostomatol Trop. 2011;34.

22. -45 .

23. Farsi N, Merdad L, Mirdad S. Caries risk assessment in preschool children in Saudi Arabia. Oral Health Prev Dent. 2013;11.

24. -280 .

25. Socioeconomic status and risk of dental caries in Japanese preschool children Tanaka K, Miyake Y, Sasaki S, Hirota Y. Socioeconomic status and risk of dental caries in Japanese preschool children.

26. the Osaka maternal. and child health study. J Public Health Dent. 2013;73:217-23.

27. Clarke M, Locker D, BerallG, Pencharz P, Kenny DJ, Judd P. Malnourishment in a population of young children with severe early childhood caries. Pediatr Dent 2006;28.

28. -9 .

29. Infant oral health

Nagarajappa R, Kakatkar G, Sharda AJ, Asawa K, Ramesh G, Sandesh N. Infant oral health.

30. Knowledge. attitude and practices of parents in Udaipur, India. Dent Res J (Isfahan). 2013;10:65965.

31. Mouradian WE, Wehr E, Crall JJ. Disparities in children's oral health and access to dental care. JAMA 2000;284.

32. -31.

33. Suresh BS, Ravishankar TL, Chaitra TR, Mohapatra AK, Gupta V. Mother's knowledge about preschool child's oral health. J Indian Soc Pedod Prev Dent 2010;28.

34. -7.

35. Szatko F, Wierzbicka M, Dybizbanska E, Struzycka I, Iwanicka-Frankowska E. Oral health of Polish three-year-olds and mothers' oral health-related knowledge. Community Dent Health 2004;21. 36. -80 .

37. Sehrawat P, Shivlingesh KK, Gupta B, Anand R, Sharma A, Chaudhry M. Oral health knowledge, awareness and associated practices of pre-school children's mothers in Greater Noida, India. Niger Postgrad Med J 2016;23.

38. -7 .

39. Blinkhorn AS, Gratrix D, Holloway PJ, Wainwright-Stringer YM, Ward SJ, Worthington HV. A cluster randomised, controlled trial of the value of dental health educators in general dental practice. $\mathrm{Br}$ Dent J 2003;195. 
40. -400 .

41. Supplementary file:.

42. File. name.

43. Supplementary. file 1.

44. Title. of data.

45. Survey.

46. Description. of data.

47. The questionnaire collected information about the (e.g., paternal education levels,child gender and order) and dental health practices (e.g., details about tooth-brushing duration and frequency, toothpaste amount used and toothbrush changing period). The questionnaire also asked about eating habits (e.g., sugar and soda intake frequency),deleterious oral habits (e.g., thumb sucking, nail and lip biting, mouth breathing,bruxism, tongue thrusting... dental visit, current caries, the way of handling orthodontic problems and early tooth extraction due to caries.

\section{Figures}

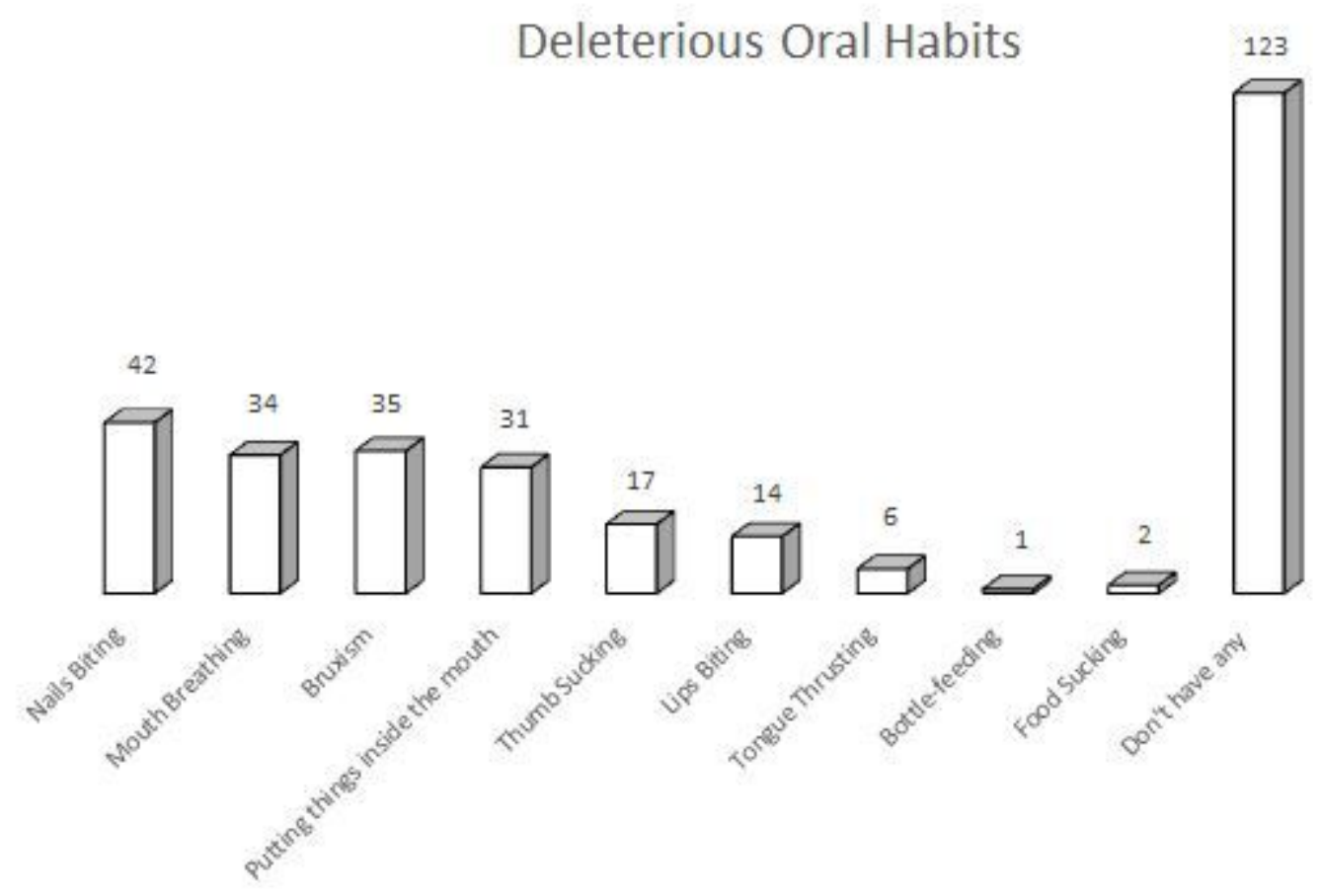

Figure 1

The frequency of deleterious oral habits.

\section{Supplementary Files}

This is a list of supplementary files associated with this preprint. Click to download. 
- Supplementaryfile1.docx

Page 23/23 